Ricardo Cordeiro ${ }^{1}$

Eduardo Rommel Olivencia Peñaloza ${ }^{1}$

Chrystiano Fonseca Cardoso 1

Daniela Barros Cortez 1

Eric Kakinami 1

Jacques José Gomes de Souza 1

Maria Tereza de Moraes Souza 1

Ricardo Augusto Fernandes 1

Rodrigo Fernando Guercia 1

Tarso Adoni 1

\section{Validade das informações ocupação e causa básica em declarações de óbito de Botucatu, São Paulo}

\author{
Validity of information on occupation \\ and principal cause on death certificates \\ in Botucatu, São Paulo
}

1 Departamento de Saúde Pública, Faculdade de Medicina de Botucatu, Universidade Estadual de São Paulo.

C. P. 543, Botucatu, SP 18618-970, Brasil.

\begin{abstract}
The aim of this paper was to evaluate the accuracy of data on death certificates for occupation and main cause of death. Measure of agreement was assessed comparing data from death certificates with those from both medical records and next-of-kin interviews, analyzing information for 552 residents of Botucatu, Southeast Brazil, who died in 1997. Kappa coefficients of 0.31 (95\% C.I. 0.29-0.34) and 0.76 (95\% C.I. 0.75-0.76) were obtained for data on occupation and main cause of death, coded by a Brazilian two-digit classification and the three-digit ICD10 classification, respectively. One can conclude that, although quality of the main cause of death is acceptable for pilot studies, data on occupation taken only from death certificates is not accurate enough to be used in epidemiological research.
\end{abstract}

Key words Death Certificates; Occupations; Underlying Cause of Death; Epidemiology

Resumo O objetivo deste trabalho é estudar a validade das informações sobre ocupação habitual e causa básica em declarações de óbito (DO) de moradores de Botucatu falecidos nesta cidade. Analisou-se a concordância dessas informações com seus padrões-ouro, estabelecidos por intermédio de entrevistas com familiares de 552 falecidos em 1997 e análise de documentação médica. O coeficiente Kappa para a concordância entre a informação sobre ocupação e o padrãoouro foi 0,31 (IC 95\% 0,29-0,34). Para a concordância entre a causa básica declarada e o padrãoouro, o coeficiente Kappa foi de 0,76 (IC 95\% 0,75-0,77). Conclui-se que, embora a validade da informação sobre a causa básica do óbito seja boa, a validade da informação sobre a ocupação habitual é bastante precária. Isto impossibilita a utilização dessas informações em estudos epidemiológicos locais. Tal constatação alerta para a necessidade de previamente verificar a fidedignidade de informações ocupacionais provenientes de declarações de óbito em estudos de saúde do trabalhador. Outra implicação desse achado é enfatizar a necessidade de investir-se na melhoria da qualidade dessas informações.

Palavras-chave Atestados de Óbito; Ocupações; Causa Básica de Morte; Epidemiologia 


\section{Introdução}

Até o final do século XIX, cada país utilizava um modelo diferente de declaração de óbito (Laurenti \& Mello-Jorge, 1994). A partir da Sexta Revisão da Classificação Estatística Internacional de Doenças (WHO, 1948), consolidou-se um modelo que passou a ser utilizado em praticamente todos os países do mundo após 1950 (Laurenti \& Mello-Jorge, 1994). Este modelo, adotado no Brasil, compõe-se de várias partes. Na parte II, Identificação do Falecido, encontra-se, entre outras informações importantes, o campo Ocupação Habitual do Falecido, que deve informar a ocupação principal do falecido, definida como a ocupação exercida por mais longo tempo durante sua vida laboral (Strauss, 1949). Na parte IV, Atestado de Óbito, devem ser declaradas as causas terminal, conseqüencial, básica e contributiva que levaram ao óbito, bem como o intervalo de tempo entre seu início e o falecimento.

Já em 1864, William Farr chamava a atenção para o potencial uso da informação sobre ocupação contida em declarações de óbito (DO) (Farr, 1984). Desde então, este documento tem subsidiado análises de mortalidade segundo ocupações em diversas partes do mundo (Office of Population Censuses and Surveys, 1970). Inferindo com base nos dados sobre a ocupação usual do falecido e as causas que o levaram ao óbito, tais análises têm contribuído para a formulação e a verificação de relações de ocorrência (Miettinen, 1985), associando exposições ocupacionais a fenômenos do processo saúde/ doença em variadas populações trabalhadoras.

Não por acaso, tais estudos baseavam-se em informações obtidas em DO. O seu caráter compulsório, aliado à sua uniformidade e universalização, tornou as informações contidas nas declarações valiosas e facilmente acessíveis.

Pesquisas dessa natureza costumam ser relativamente simples e baratas, sendo particularmente úteis na vigilância de óbitos de grupos ocupacionais para os quais é difícil organizar e gerenciar coortes, como trabalhadores de pequenas indústrias e certos grupos ocupacionais não sindicalizados (Dubrow et al., 1987). Entretanto, a validade das informações é uma grande preocupação nesses estudos.

Com relação à validação dos dados sobre ocupação contidos nas DO, ainda não há estudos analisando a situação no Brasil publicados em periódicos de circulação nacional. Porém, no âmbito internacional, há um grande número de trabalhos abordando essa questão (Buechley et al., 1956; Alderson, 1972; Wegman \& Peters, 1978; Milhan, 1983; Swanson et al., 1984; Steen- land \& Beaumont, 1984; Balarajan, 1985; Gute \& Fulton, 1985; Schumacher, 1986; Turner et al., 1987; Schade \& Swanson, 1988; Olsen et al., 1990). Tais estudos, por intermédio de métodos diferentes e complementares, concluem ser geralmente insatisfatória a concordância entre a ocupação referida na DO e aquela de fato exercida pelo trabalhador durante a maior parte de sua vida. Com relação à validação dos dados sobre as causas do óbito assinaladas nas DO, a situação nacional já é um pouco conhecida. Há estudos pontuais em algumas localidades brasileiras (Milanesi \& Laurenti, 1964; Fonseca \& Laurenti, 1974; Schinitman, 1990; Mello-Jorge, 1990) apontando um grau de concordância simples, que varia de $62 \%$ a $72 \%$, entre a informação sobre a causa básica do óbito referida na DO e aquela que de fato levou ao óbito.

O objetivo deste trabalho é estudar a validade das informações sobre a ocupação habitual do falecido e a causa básica do óbito em DO de moradores de Botucatu, visando contribuir para a melhoria de seu preenchimento e fornecer subsídios aos pesquisadores que planejam estudos epidemiológicos no campo da saúde do trabalhador, utilizando informações obtidas em declarações de óbito em nosso meio.

\section{Material e método}

Analisou-se a validade das informações do campo Ocupação Habitual do Falecido e da causa básica do óbito em DO dos moradores de Botucatu com idade igual ou superior a dez anos, falecidos entre 01/01/97 e 31/12/97. O acesso às DO foi obtido por intermédio de um acordo estabelecido com as três empresas funerárias que operam na cidade. Estas forneceram, durante o período da pesquisa, uma cópia de cada DO, que era recebida pela equipe de pesquisa entre 24 e 72 horas após o sepultamento.

Logo no seu recebimento, a causa básica do óbito era definida com base na aplicação das regras de seleção estabelecidas na Décima Revisão da Classificação Internacional de Doenças (OMS, 1994). Tal seleção era feita por um profissional da equipe de pesquisa com grande experiência em codificação de mortalidade. A causa básica assim selecionada será chamada causa básica DO. Analogamente, a informação referida na DO no campo Ocupação Habitual do Falecido será chamada ocupação habitual DO.

Definiu-se a validade das informações causa básica DO e ocupação habitual DO como os seus graus de correção, isto é, o quanto estas informações expressam a real causa básica e ocupação habitual para a população estudada. 
Ela foi determinada medindo-se a concordância dessas informações com seus respectivos padrões-ouro, após codificação.

O padrão-ouro para a causa básica DO foi construído de acordo com o seguinte processo. Antes do início da coleta de dados para este estudo, solicitou-se formalmente aos diretores clínicos dos quatro hospitais de Botucatu permissão para acessar e analisar os prontuários hospitalares de todos os óbitos a ocorrer nesses hospitais durante o ano de 1997, obtendose a autorização solicitada. Também foi solicitada formalmente autorização para consulta de todos os laudos emitidos em 1997 pelo Serviço de Verificação de Óbitos da Faculdade de Medicina de Botucatu - Universidade Estadual Paulista (UNESP) e pelo Instituto Médico Legal do município, obtendo-se permissão de seus respectivos responsáveis.

Durante o ano de 1997, duas a três semanas após o óbito, um entrevistador visitava as famílias dos falecidos e entrevistava um familiar adulto destes, geralmente filho(a), cônjuge, irmão(ã) ou pai/mãe. Nessas entrevistas, era recuperada a história do óbito e os problemas relevantes de saúde dos falecidos, segundo o respondente. Paralelamente, para os óbitos hospitalares, era pesquisado o desenvolvimento do processo que levou à morte, por intermédio do estudo do prontuário do falecido, que abrangia, inclusive, a análise de exames subsidiários importantes para o estabelecimento de diagnósticos, como, por exemplo, laudos de biópsia e de exames de imagens. A análise dos prontuários foi feita por um médico especialista em saúde ocupacional, membro da equipe de pesquisa.

Para os óbitos examinados pelo Serviço de Verificação de Óbitos, pesquisou-se ainda o laudo emitido por este serviço. Também nos casos de suspeita de falecimento por morte violenta, foram analisados os laudos emitidos pelo Instituto Médico Legal. Em muitas ocasiões, para complemento de informações, o profissional médico que preencheu o atestado de óbito, ou o que procedeu à necropsia, foi entrevistado. Quanto aos óbitos domiciliares de pacientes crônicos com prontuários hospitalares, localizaram-se e analisaram-se tais prontuários, sempre que possível, segundo o mesmo procedimento acima descrito. Em alguns casos, quando se julgou necessário, foram localizados e analisados prontuários ambulatoriais de pacientes crônicos em seguimento ambulatorial regular.

Ao final desse processo, de posse de todas as informações obtidas, o atestado de óbito (parte IV da DO) era reconstruído caso a caso por um membro da equipe de pesquisa com grande experiência em codificação de mortalidade. Assim, com base no documento reconstruído e de acordo com as regras de seleção da Décima Revisão da Classificação Internacional de Doenças (CID-10) (OMS, 1994), o mesmo pesquisador selecionava a causa básica do óbito, sendo esta denominada causa básica padrão-ouro.

O padrão-ouro para a ocupação habitual DO foi construído da seguinte maneira. No momento da entrevista com familiar, era também recuperada a história ocupacional do falecido, a partir de seus dez anos de idade e até a última ocupação exercida, anotando-se pormenorizadamente as ocupações e os seus respectivos tempos de exercício. Sempre que disponível, a carteira profissional do morto era consultada, ratificando-se e complementando-se as informações obtidas. Para cada um dos falecidos, definiu-se a ocupação habitual como aquela exercida por mais tempo, sendo esta informação considerada o padrão-ouro e denominada neste texto ocupação habitual padrão-ouro. Em caso de duas ou mais ocupações diferentes terem sido exercidas pelo mesmo tempo, considerou-se a habitual aquela exercida mais recentemente. Para indivíduos que nunca exerceram uma ocupação, considerou-se padrão-ouro a informação "sem ocupação". Cerca de 10\% dos familiares entrevistados, escolhidos ao acaso, foram revisitados por coordenadores de campo, que reaplicavam parcialmente a entrevista. Os dados eram comparados com as entrevistas originais para checar sua qualidade, visando identificar eventuais imprecisões, erros sistemáticos ou mesmo fraudes, os quais não foram identificados.

A validade da causa básica DO foi determinada medindo-se o grau de concordância desta informação com a causa básica padrão-ouro, após ambas terem sido codificadas ao nível do terceiro dígito da CID-10 (OMS, 1994). A concordância foi medida por intermédio do coeficiente Kappa, função da freqüência de pares concordantes em classificações independentes segundo variáveis categóricas exclusivas e exaustivas, corrigidas as concordâncias devidas ao acaso (Cohen, 1960).

De modo análogo, determinou-se a validade da ocupação habitual DO medindo-se também por intermédio do coeficiente Kappa (Cohen, 1960) - o grau de concordância daquela informação com a ocupação habitual padrão-ouro, após ambas serem codificadas de acordo com a classificação de ocupações de Rumel (1987). Tal classificação é uma simplificação que agrupa ocupações da Classificação Brasileira de Ocupações (Ministério do Traba- 
lho, 1982) com base na classificação de ocupações utilizada pelo IBGE - Fundação Instituto Brasileiro de Geografia e Estatística (1983).

\section{Resultados}

Durante o ano de 1997, faleceram 635 moradores em Botucatu. A estimativa do tamanho da população no município em 1o de julho de 1997 era de 102.494 habitantes (IC 95\% 101.810 - 103.179) habitantes (Cordeiro et al., 1999). Deste modo, o coeficiente geral de mortalidade em Botucatu foi de 6,20 óbitos/ 1000 habitantes (IC 95\% 6,15-6,24), sendo o coeficiente de mortalidade padronizado igual a 4,97 óbitos / 1000 habitantes (IC 95\% 4,94-5,00), tomando-se como referência a população do Brasil em 1996 (IBGE, 1997).

\section{a) Validade do campo Ocupação Habitual do Falecido}

Dentre os 635 moradores falecidos, 603 tinham idade igual ou superior a dez anos no momento do óbito. Destes, conseguiu-se recuperar, por meio de entrevista com familiar, a história ocupacional de 549 (91,0\%) deles. As 54 perdas $(9,0 \%)$ distribuíram-se como segue: as famílias de 28 (4,6\%) falecidos não foram localizadas; as de nove $(1,5 \%)$ não souberam informar suas ocupações habituais e as de 11 (1,8\%) recusaram-se a informar. Além disso, seis falecidos (1,0\%), que viviam em asilos ou em um hospital psiquiátrico, não tinham família. A distribuição de sexo, idade e estado civil destes falecidos não diferiu significativamente da distribuição destas características entre os demais 549, exceto para os seis moradores que faleceram sem deixar familiares, todos bastante idosos.

A Tabela 1 apresenta a situação do preenchimento do campo Ocupação Habitual do Falecido nas 549 DO. Observou-se que apenas $41,3 \%$ destas traziam este campo preenchido com uma ocupação de fato. Em 15,7\% das vezes o campo trazia informações imprecisas, impossibilitando a identificação da ocupação habitual exercida, como, por exemplo, “ajudante”, “auxiliar”, "funcionário público”, "servidor municipal" "militar", "autônomo" etc. Em 0,5\% das vezes este campo esteve em branco. A freqüência de informações não relacionadas à ocupação "pensionista”, "menor", “desempregado”, “aposentado", "estudante", "do lar" - foi de 42,5\%.

A concordância simples entre as informações ocupação habitual DO e ocupação habitual padrão-ouro, codificadas segundo a classificação de Rumel (1987), foi de 37,7\%. Para este cálculo, interpretou-se a informação "do lar", segundo o pensamento corrente no município, como significando indivíduo que nunca exerceu atividade profissional, sendo classificado como subgrupo 601 na classificação de Rumel (1987). A esta concordância correspondeu um coeficiente Kappa de 0,31 (IC 95\% 0,29-0,34). A Tabela 2 apresenta a concordância simples e o coeficiente Kappa para a informação sobre ocupação nas 549 DO agrupadas segundo sexo (masculino e feminino) e idade (50\% mais velhos e $50 \%$ mais novos), bem como para o grupo dos $50 \%$ mais velhos do sexo masculino.

\section{b) Validade da Causa Básica do Óbito}

A Tabela 3 apresenta a distribuição dos óbitos dos 603 moradores de Botucatu com idade igual ou superior a dez anos em 1997, de acordo com o local de ocorrência. Nela, observa-se que cerca de dois terços dos falecimentos (390) ocorreram em hospitais, tendo o terço restante ocorrido, em sua maioria, em casa (173) e uma pequena parte em locais públicos (40), tais como ruas, praças, rodovias, bares, rios, lagos etc.

Existem, em Botucatu, quatro hospitais, sendo um universitário público (Hospital das Clínicas da Faculdade de Medicina de Botucatu - UNESP), um psiquiátrico estadual, e dois hospitais gerais particulares, tendo a maioria dos óbitos hospitalares (249) ocorrido no hospital universitário. Deste total, foram localizados e examinados 199 prontuários, não se conseguindo encontrar cinqüenta deles. Para os restantes 141 óbitos ocorridos nos outros três hospitais não universitários do município, conseguiu-se localizar e analisar todos os respectivos prontuários. Sendo assim, do total de 390 óbitos hospitalares, foram localizados e examinados 340 prontuários.

Para 26 dos 173 óbitos domiciliares ocorridos, conseguiu-se encontrar prontuários de serviços ambulatoriais do Hospital das Clínicas da Faculdade de Medicina de Botucatu, ou de internações anteriores em suas enfermarias ou pronto-socorro, contendo informações suficientes para o estabelecimento do processo básico que levou ao óbito.

Dos quarenta óbitos ocorridos em locais públicos, em sua maioria decorrentes de causas externas, não se conseguiu localizar nenhum prontuário ambulatorial ou hospitalar contendo informações suficientes.

Foi feita necropsia em 207 dos 603 moradores falecidos em Botucatu. O Serviço de Verificação de Óbitos do Departamento de Patologia da Faculdade de Medicina de Botucatu realizou necropsia em 136 deles, para esclare- 
cimento de óbitos inicialmente mal definidos. O Instituto Médico Legal do município procedeu ao exame em 71, para esclarecer óbitos considerados suspeitos de serem decorrentes de causas externas. Todos os 207 laudos destes exames foram localizados e examinados, e, para esclarecimento da evolução dos processos que levaram parte desses 603 moradores ao óbito, foram realizadas 129 entrevistas com médicos que deram assistência a esses falecidos.

As Figuras 1, 2 e 3 são diagramas de Venn, que mostram como se interseccionam os conjuntos de óbitos documentados por prontuários hospitalares, prontuários ambulatoriais, necropsia do Serviço de Verificação de Óbitos (SVO), necropsia do Instituto Médico Legal (IML) e entrevistas com médicos assistentes, nos universos de óbitos hospitalares, domiciliares e em locais públicos, respectivamente. Com base na análise dessas figuras, verifica-se que, dos 603 que faleceram dentro da faixa etária de interesse, 552 (91,5\%) indivíduos tiveram a história do óbito estabelecida por, no mínimo, um dos seguintes meios: prontuário médico, laudo de necropsia, entrevista com médico assistente. Para estes óbitos, a causa básica padrão-ouro foi definida valendo-se desta documentação, conforme descrito anteriormente. Para os restantes $51(8,5 \%)$ óbitos, não se conseguiu nenhuma documentação médica, sendo estes excluídos da análise da validade da causa básica DO.

A concordância simples entre as informações causa básica DO e causa básica padrãoouro, codificadas segundo a Lista de Categorias de Três Caracteres da CID-10 (OMS, 1994), foi de $83,1 \%$. A esta concordância correspondeu um coeficiente Kappa de 0,76 (IC 95\% 0,75-0,77). A Tabela 4 apresenta a concordância simples e o coeficiente Kappa acima especificados para estas 552 DO agrupadas segundo sexo (masculino e feminino) e segundo a idade (50\% mais velhos e $50 \%$ mais novos), bem como para o grupo dos $50 \%$ mais velhos do sexo feminino. Tal concordância não variou significativamente quando se classificaram os moradores falecidos de acordo com o grupo ocupacional principal (Rumel, 1987) a que pertenciam, isto é, não se encontrou associação entre grupo ocupacional e validade da causa básica.

\section{Discussão}

É bastante insatisfatória a validade da informação contida no campo Ocupação Habitual do Falecido nas DO analisadas. Conforme observado na Tabela 2, o coeficiente Kappa de con-

Tabela 1

Distribuição das informações do campo ocupação habitual do falecido de acordo com seu conteúdo nas DO analisadas. Botucatu, 1997.

\begin{tabular}{lcc}
\hline Conteúdo do campo & $\mathbf{n}$ & Freqüência (\%) \\
\hline Ocupação bem definida & 227 & 41,3 \\
& & 15,7 \\
Informação ocupacional imprecisa & 86 & 0,5 \\
Em branco & 3 & \\
Informação ocupacional inválida & 233 & 42,5 \\
pensionista & 3 & 0,5 \\
menor & 1 & 0,2 \\
desempregado & 2 & 0,4 \\
aposentado & 46 & 8,4 \\
estudante & 6 & 1,1 \\
do lar & 175 & 31,9 \\
Total & 549 & 100,0 \\
\hline
\end{tabular}

Tabela 2

Concordância simples e coeficiente Kappa para ocupação habitual DO e ocupação habitual padrão-ouro nas DO analisadas. Botucatu, 1997.

\begin{tabular}{lcccc}
\hline População & $\mathbf{n}$ & $\begin{array}{l}\text { Concordância } \\
\text { simples (\%) }\end{array}$ & $\begin{array}{l}\text { Coeficiente } \\
\text { Kappa }\end{array}$ & IC 95\% Kappa \\
\hline Total & 549 & 37,7 & 0,31 & $0,29-0,34$ \\
Sexo masculino & 313 & 39,6 & 0,35 & $0,32-0,38$ \\
Sexo feminino & 236 & 35,2 & 0,13 & $0,08-0,17$ \\
$50 \%$ mais velhos & 275 & 44,4 & 0,36 & $0,32-0,40$ \\
$50 \%$ mais jovens & 274 & 30,3 & 0,25 & $0,22-0,28$ \\
$50 \%$ mais velhos masculinos & 131 & 49,6 & 0,43 & $0,37-0,48$ \\
\hline
\end{tabular}


Tabela 3

Distribuição dos 603 óbitos estudados segundo local

de ocorrência. Botucatu, 1997.

\begin{tabular}{lcc}
\hline Local de ocorrência & $\mathbf{n}$ & $\begin{array}{c}\text { Freqüência } \\
\text { relativa (\%) }\end{array}$ \\
\hline Hospital & 390 & 64,7 \\
Domicílio & 173 & 28,7 \\
Locais públicos & 40 & 6,6 \\
Total & 603 & 100,0 \\
\hline
\end{tabular}

Figura 1

Distribuição dos 390 óbitos hospitalares segundo documentação obtida.

Botucatu, 1997

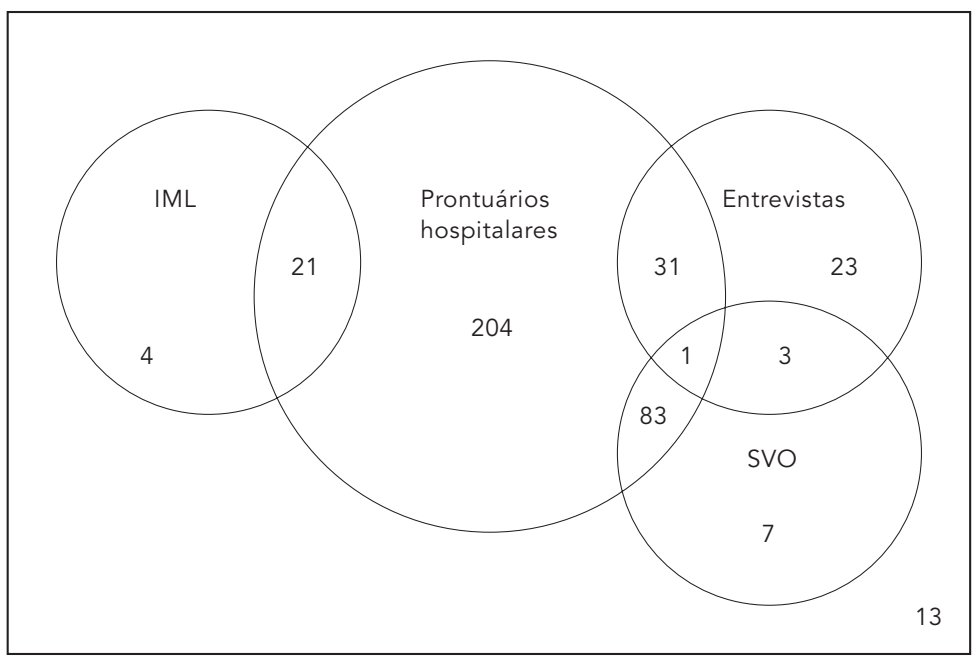

cordância entre a ocupação habitual DO e a ocupação habitual padrão-ouro no total de 549 DO analisadas foi de apenas 0,31 (IC 95\% 0,29$0,34)$, com discreto aumento associado ao sexo masculino e idade maior que a mediana. Não é possível situar este resultado no âmbito nacional, uma vez que não foram ainda publicados, em periódicos de circulação nacional, estudos de validação dessa informação em DO de localidades brasileiras. No entanto, há um grande número de estudos dessa natureza em outros países, o que, de certa forma, permite dimensionar a situação de Botucatu em relação a outras localidades.

Já em 1956, Buechley et al., estudando 423 óbitos causados por câncer, ocorridos entre 1948 e 1952 no Estado da Califórnia, Estados Unidos, verificaram ser de $51 \%$ a concordância simples entre a ocupação habitual referida nas DO destes óbitos e o padrão-ouro, após ambas terem sido codificadas segundo uma classificação de três dígitos (U.S. Bureau of the Census, 1950).

Alderson (1972) amostrou sistematicamente três quartos dos óbitos ocorridos entre indivíduos do sexo masculino com idade entre vinte e 64 anos na cidade de Bristol, Inglaterra, entre 1962 e 1963. De modo análogo a este trabalho, entre duas a quatro semanas após o óbito, entrevistaram-se as famílias dos falecidos, recuperando-se a história ocupacional dos mesmos. Foram estudados 591 óbitos, encontrando-se uma concordância simples de $79 \%$ entre a ocupação habitual referida na DO e o padrão-ouro, após codificadas segundo uma classificação de três dígitos.

Swanson et al. (1984) analisaram registros de 30.194 casos de câncer incidentes diagnosticados entre os anos de 1973 e 1979, na região metropolitana de Detroit, Estados Unidos, e registrados na Cancer Surveillance Section of the Division of Epidemiolgy of the Michigan Cancer Foundation (Swanson \& Brennan, 1981). Dentre estes casos, foram identificados 352 óbitos ocorridos em 1982, para os quais havia registro da história ocupacional obtida por intermédio de entrevistas com os próprios pacientes. Os autores encontraram uma concordância simples de $76,1 \%$ entre a ocupação habitual referida na DO e aquela obtida com os pacientes em vida, após codificadas segundo uma classificação de três dígitos (U.S. Bureau of the Census, 1981).

Steenland \& Beaumont (1984) estudaram 2.198 óbitos de indivíduos pertencentes a coortes que foram acompanhadas em 11 diferentes estudos norte-americanos, para os quais havia um padrão-ouro para a ocupação. Estes autores encontraram uma concordância simples de $64,7 \%$ entre a ocupação habitual referida na DO e o padrão-ouro, após codificadas segundo uma classificação de ocupações de três dígitos (U.S. Bureau of the Census, 1981). Essa concordância associava-se com a raça (maior em brancos) e o sexo (maior em homens) dos trabalhadores.

Gute \& Fulton (1985) estudaram 446 óbitos ocorridos entre 1962 e 1981 na cidade de Providence, Estados Unidos. Esses indivíduos haviam participado de um estudo em 1962, tendo sido entrevistados a respeito de sua história ocupacional. Os autores encontraram uma concordância simples de $66 \%$ entre a ocupação habitual referida na DO e o padrão-ouro obtido com os próprios trabalhadores em vida, após codificadas segundo uma ocupação de três dígitos (Office of Population Censuses and Surveys, 1970). 
Balarajan (1985) estudou 704 óbitos por câncer ocorridos entre 1981 e 1982 na Inglaterra, os quais eram parte de uma pesquisa casocontrole com trabalhadores da agricultura, sendo os casos sarcomas de tecidos moles, e os controles outros tipos de câncer. Os trabalhadores em questão haviam sido entrevistados quanto à sua história ocupacional ao entrarem no estudo. $\mathrm{O}$ autor encontrou uma concordância simples de 73,4\% entre a ocupação habitual referida na DO e a ocupação principal referida pelo trabalhador em vida, após codificadas segundo uma classificação de três dígitos (Office of Population Censuses and Surveys, 1970).

Turner et al. (1987) estudaram 87 óbitos de moradores do Estado de Utah, Estados Unidos, com câncer de bexiga diagnosticado entre 1978 e 1982. Esses pacientes faziam parte de um estudo caso-controle e haviam sido entrevistados em vida a respeito de sua história ocupacional. Os autores encontraram uma concordância simples de 54\% entre a ocupação habitual referida na DO e a ocupação principal referida em vida, após codificadas segundo uma classificação de três dígitos (U.S. Bureau of the Census, 1981).

Schade \& Swanson (1988) estudaram 2.435 óbitos por câncer ocorridos na região metropolitana de Detroit, Estados Unidos, entre 1984 e 1986. A concordância simples encontrada entre o padrão-ouro, construído com base em entrevistas com familiares, e a ocupação habitual referida na DO foi de $47,9 \%$, após codificação segundo uma classificação de ocupações de três dígitos (U.S. Bureau of the Census, 1981). Os autores referem que essa concordância variava significativamente de acordo com as seguintes covariáveis: raça (maior para brancos), sexo (maior para mulheres), estado civil (maior para casados), tempo de trabalho (maior para mais antigos na profissão) e idade no diagnóstico (maior para os mais velhos).

Olsen et al. (1990) estudaram 1.399 óbitos de trabalhadores de uma grande indústria química nos Estados Unidos, encontrando uma concordância simples variando de $50 \%$ a $70 \%$ entre o padrão-ouro, registro da empresa, e a ocupação habitual referida na DO, após codificação segundo uma classificação de três dígitos (U.S. Bureau of the Census, 1981). A concordância se associou com o tempo de trabalho, sendo maior para os mais antigos na empresa.

Deve-se levar em consideração que todas essas análises de concordância foram feitas após as ocupações terem sido codificadas de acordo com classificações de três dígitos, consideravelmente mais pormenorizadas do que a utilizada neste trabalho, a classificação de Ru-
Figura 2

Distribuição dos 173 óbitos domiciliares segundo documentação obtida Botucatu, 1997.

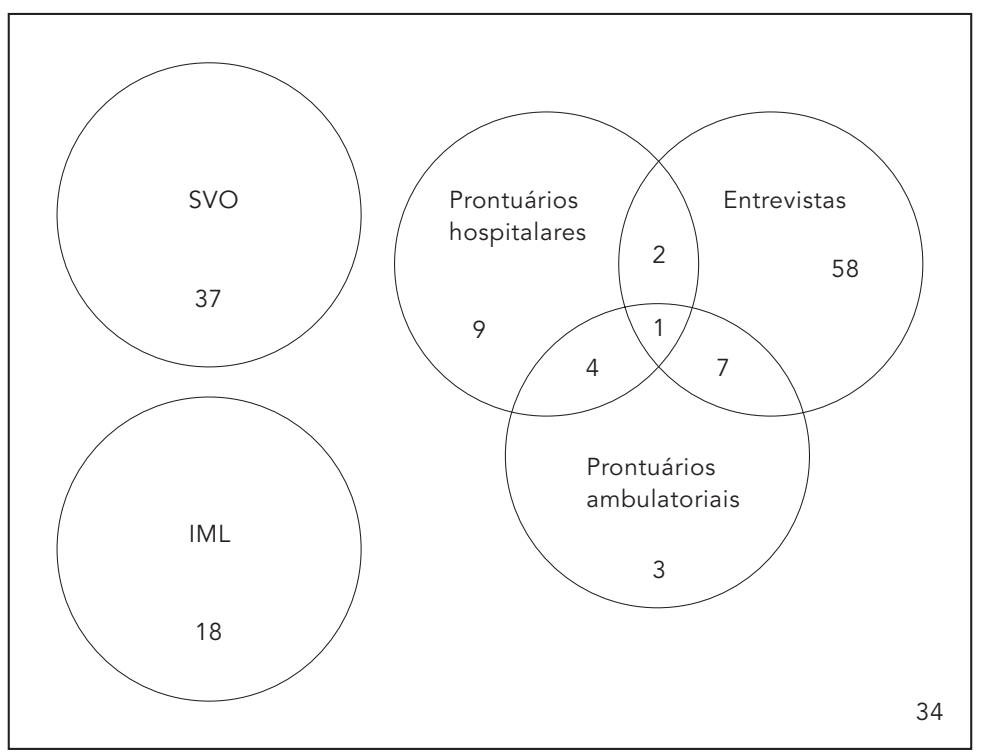

Figura 3

Distribuição dos 40 óbitos ocorridos em vias públicas segundo documentação obtida, Botucatu, 1997.

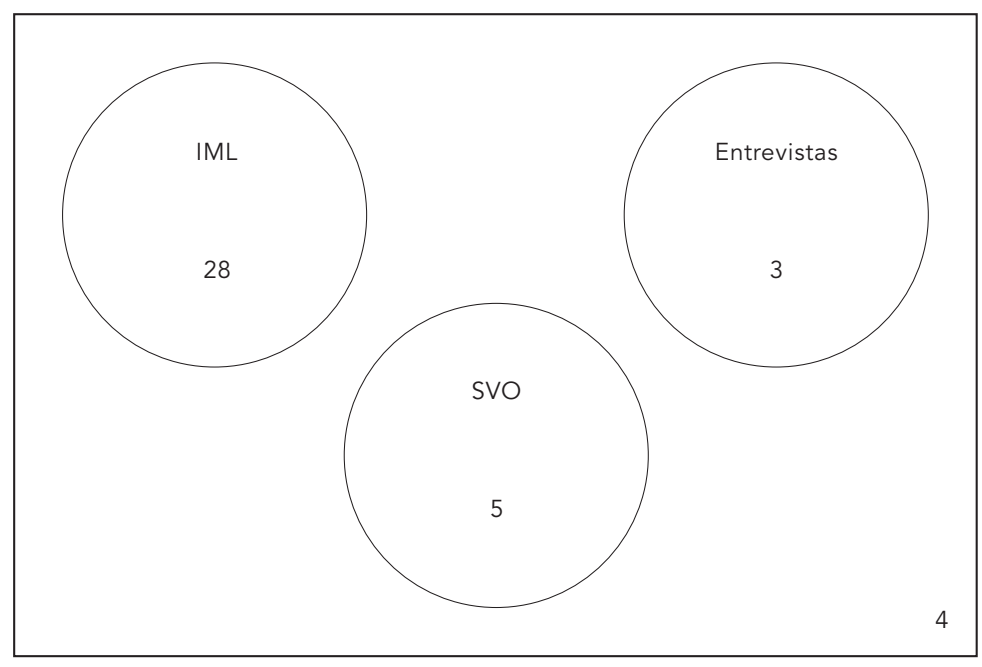


Concordância simples e coeficiente Kappa para causa básica habitual DO e causa básica padrão-ouro nas DO analisadas. Botucatu, 1997

\begin{tabular}{lcccc}
\hline População & $\mathbf{n}$ & $\begin{array}{l}\text { Concordância } \\
\text { simples (\%) }\end{array}$ & $\begin{array}{l}\text { Coeficiente } \\
\text { Kappa }\end{array}$ & IC 95\% Kappa \\
\hline Total & 552 & 83,1 & 0,76 & $0,75-0,77$ \\
Sexo masculino & 317 & 80,5 & 0,75 & $0,73-0,77$ \\
Sexo feminino & 235 & 86,6 & 0,81 & $0,78-0,84$ \\
$50 \%$ mais velhos & 276 & 84,4 & 0,78 & $0,75-0,80$ \\
$50 \%$ mais jovens & 276 & 81,9 & 0,77 & $0,75-0,79$ \\
$50 \%$ mais velhos masculinos & 121 & 87,5 & 0,81 & $0,78-0,84$ \\
\hline
\end{tabular}

mel (1987), que apresenta apenas 16 categorias. Isto torna conservadora - favorecendo a hipótese de igualdade - a comparação aqui feita entre os resultados obtidos em Botucatu e os da literatura internacional supracitados.

Diferentemente do que ocorre quanto à validade da informação Ocupação Habitual do Falecido, é relativamente boa a validade da informação Causa Básica da DO no conjunto analisado. Conforme a Tabela 4, a concordância simples entre a causa básica DO e a causa básica padrão-ouro, após codificadas de acordo com a CID-10 (OMS, 1994), foi de 83,1\%, implicando um coeficiente Kappa de 0,76 (IC 95\% 0,75-0,77). Essa concordância simples variou de $80,5 \%$ a $87,5 \%$, com o coeficiente Kappa oscilando entre 0,75 (IC 95\% 0,73-0,77) e 0,81 (IC 95\% 0,78-0,84), de acordo com as covariáveis sexo e idade dos falecidos. Esses resultados se destacam positivamente quando comparados com os apresentados por outros autores que estudaram a situação nacional.

Já em 1964, Milanesi \& Laurenti estudaram uma amostra aleatória de 534 DO de moradores da cidade de São Paulo, falecidos nos meses de fevereiro e março dos anos de 1962 e 1963, com idades entre 15 e 74 anos. O padrão-ouro para a causa básica destes óbitos foi construído valendo-se de entrevistas com familiares, com médicos assistentes e análise de documentação médica. Os autores referem que, em $67,6 \%$ das DO, pôde ser encontrada, em alguma localização do atestado de óbito, a causa básica padrão-ouro (Milanesi \& Laurenti, 1964).

Fonseca \& Laurenti (1974) estudaram uma amostra de 1.895 óbitos ocorridos em hospitais e pronto-socorros da cidade de São Paulo entre 1971 e 1972. Os autores encontraram uma concordância simples de $62,1 \%$ entre o padrão-ouro, construído com base na análise de prontuários hospitalares, e a causa básica DO, após se- rem codificadas segundo a lista de categorias de três algarismos da Oitava Revisão da Classificação Internacional de Doenças (OMS, 1957).

Schnitman (1990) estudou a validade da informação Causa Básica de moradores de Salvador que faleceram de câncer no ano de 1983. Para tanto, amostrou DO cujas causas básicas selecionadas eram tumores de estômago, pulmão, fígado, pâncreas, bexiga, além de tumores de localização não especificada e leucemias. A autora encontrou uma concordância simples de $65 \%$ entre a causa básica DO e a causa básica padrão-ouro, após codificação de ambas segundo a lista de categorias de três algarismos da Nona Revisão da Classificação Internacional de Doenças (OMS, 1985). O padrão-ouro foi construído por meio de análises de prontuários hospitalares desses pacientes.

Mello-Jorge (1990) estudou mortes violentas atingindo menores de 15 anos ocorridas na cidade de São Paulo em 1985. Utilizando um padrão-ouro construído de entrevistas domiciliares, hospitalares, em delegacias de polícia e no IML de São Paulo, encontrou uma concordância simples de 71,6\% entre este padrão e a causa básica selecionada nas DO, após codificação segundo a lista de categorias de três algarismos da Nona Revisão da Classificação Internacional de Doenças (OMS, 1985).

\section{Conclusões}

Embora seja boa a validade da informação Causa Básica do Óbito, a validade da informação Ocupação Habitual do Falecido é bastante precária nas DO de Botucatu, o que impossibilita a utilização dessas informações em estudos epidemiológicos locais. Além disso, tal constatação alerta para a necessidade de previamente verificar-se a fidedignidade de informações 
ocupacionais provenientes de DO em estudos de saúde do trabalhador que utilizam essa fonte de informação e que tenham sido realizados em outras localidades brasileiras. Outra implicação desse achado é enfatizar a necessidade de investir-se na melhoria da qualidade dessas informações nas declarações de óbito, que, infelizmente, é o único documento a abranger, longitudinalmente, a totalidade dos trabalhadores brasileiros.

\section{Agradecimentos}

Agradecemos a Mônica Aparecida Silveira Kron, pela orientação e supervisão na codificação das causas de óbito e seleção das causas básicas. Agradecemos também à Fundação de Amparo à Pesquisa do Estado de São Paulo e ao Conselho Nacional de Desenvolvimento Científico e Tecnológico pelo financiamento deste estudo (processos Fapesp 96/7583-4, 96/7584-0, 96/7585-7, 97/12782-9, 97/12783-5, 97/12784-1; e CNPq 520605/96-9).

\section{Referências}

ALDERSON, M. R., 1972. Some sources of error in British occupational mortality data. British Journal of Industrial Medicine, 29:245-254.

BALARAJAN, R., 1985. Comparison of occupations recorded at cancer registration and death. Public Health (London), 99:169-173.

BUECHLEY, R.; DUNN, J. E.; LINDEN, G. \& BRESLOW, L., 1956. Death certificate statement of occupation: Its usefulness in comparing mortalities. Public Health Reports, 71:1105-1111.

COHEN, J., 1960. A coefficient of agreement for nominal scales. Educational and Psychological Measurement, 20:37-46.

CORDEIRO, R.; PEÑALOZA, E. R. O.; CARDOSO, C. F.; CORTEZ, D. B.; KAKINAMI, E.; SOUZA, J. J. G.; SOUZA, M. T. M.; FERNANDES, R. A.; GUERCIA, R. F. \& ADONI, T., 1999. Estimativa da perda de heterogeneidade decorrente do uso de amostragem de conglomerados para a população economicamente ativa - $\mathrm{O}$ efeito do desenho. (mimeo).

DUBROW, R.; SESTITO, J. P.; LALICH, N. R.; BURNETT, C. A. \& SALG, J. A., 1987. Death certificatebased occupational mortality surveillance in the United States. American Journal of Industrial Medicine, 11:329-342.

FARR, W., 1984. Letter to the Registrar-General on the mortality in the registration districts of England during the 10 years 1851-60. Supplement to the 25th Annual Report of the Registrar General of Births, Deaths and Marriages in England, pp. xxxv, London: Her Majesty Stationery Office.
FONSECA, L. A. M. \& LAURENTI, R., 1974. A qualidade da certificação médica da causa de morte em São Paulo, Brasil. Revista de Saúde Pública, 8:21-29.

GUTE, S. M. \& FULTON, J. P., 1985. Agreement of occupation and industry data on Rhode Island death certificate with two alternative sources of information. Public Health Reports, 100:65-72.

IBGE (Fundação Instituto Brasileiro de Geografia e Estatística), 1983. Censo Demográfico: Mão de Obra; São Paulo. Vol. 1, T. 5, no 19. Rio de Janeiro: IBGE.

IBGE (Fundação Instituto Brasileiro de Geografia e Estatística), 1997. Contagem da População, 1996. Vol. 1. Rio de Janeiro: IBGE.

LAURENTI, R. \& MELLO-JORGE, M. H. P., 1994. O Atestado de Óbito. São Paulo: Centro da Organização Mundial da Saúde para Classificação de Doenças em Português.

MELLO-JORGE, M. H. P., 1990. Situação atual das estatísticas oficiais relativas à mortalidade por causas externas. Revista de Saúde Pública, 24:217-223.

MIETTINEN, O. S., 1985. Theoretical EpidemiologyPrinciples of Occurrence Research in Medicine. New York: John Wiley \& Sons.

MILANESI, M. L. \& LAURENTI, R., 1964. Estado atual da certificação médica da causa de óbito. Revista da Associação Médica Brasileira, 10:111-116.

MILHAN, S., 1983. Occupational Mortality in Washington State 1950 - 1979. Publication No. 83-116. Cincinati: National Institute for Occupational Safety and Health DHHS/NIOSH. 
MINISTÉRIO DO TRABALHO, 1982. Sistema Nacional de Emprego - Classificação Brasileira de Ocupações. Brasília: Ministério do Trabalho.

OFFICE OF POPULATION CENSUSES AND SURVEYS, 1970. Classification of Occupations. London: Her Majesty Stationery Office.

OLSEN, G. W.; BRONDUM, J.; BODNER, K. M.; KRAVAT, B. A.; MANDEL, J. S.; MANDEL, J. H. \& BOND, G. G., 1990. Occupation and industry on death certificates of long-term chemical workers: Concordance with work history records. American Journal of Industrial Medicine, 17:465-481.

OMS (Organización Mundial de la Salud), 1957. Manual de la Clasificación Estadística Internacional de Enfermedades, Traumatismos y Causas de Defunción. Ginebra: OMS.

OMS (Organização Mundial da Saúde), 1985. Manual da Classificação Estatística Internacional de Doenças, Lesões e Causas de Óbito. 9a Revisão, Vol. 1. São Paulo: Centro Brasileiro para Classificação de Doenças.

OMS (Organização Mundial de Saúde), 1994. CID-10 Classificação Estatística Internacional de Doenças e Problemas Relacionados à Saúde - Décima Revisão. São Paulo: Edusp.

RUMEL, D., 1987. Indicadores de Mortalidade por Categoria Ocupacional e Nível Social. Dissertação de Mestrado, São Paulo: Faculdade de Saúde Pública, Universidade de São Paulo.

SCHADE, W. J. \& SWANSON, M., 1988. Comparison of death certificate occupation and industry data with lifetime occupational histories obtained by interview: Variations in the accuracy of death certificate entries. American Journal of Industrial Medicine, 14:121-136.

SCHNITMAN, A., 1990. Análise da fidedignidade da declaração da causa básica de morte por câncer em Salvador, Brasil. Revista de Saúde Pública, 24:490-496.

SCHUMACHER, M. C., 1986. Comparison of occupation and industry information from death certificates and interviews. American Journal of Public Health, 76:635-637.
STEENLAND, K. \& BEAUMONT, J., 1984. The accuracy of occupation and industry data on death certificates. Journal of Occupational Medicine, 26:288-296.

STRAUSS, R. C., 1949. Guide for Reporting Occupation and Industry on Death Certificates. Washington, D.C.: U.S. Government Printing Office.

SWANSON, G. M. \& BRENNAN, M. J., 1981. Cancer incidence and mortality in metropolitan Detroit, 1973-1977. In: Surveillance, Epidemiology, and End Results: Incidence and Mortality Data, 19731977 (J. L. Young, C. L. Percy \& A. J. Asire, eds.), National Institutes of Health, National Cancer Institute Monograph 57, pp.17-26, Washington, D.C.: U.S. Government Printing Office.

SWANSON, G. M.; SCHWARTZ, A. G. \& BURROWS, R. W., 1984. An assessment of occupational and industry data from death certificates and hospital medical records for population-based surveillance. American Journal of Public Health, 74:464467.

TURNER, D. W.; SCHUMACHER, M. C. \&WEST, D. W. 1987. Comparison of occupational interview data to death certificate data in Utah. American Journal of Industrial Medicine, 12:145-151.

UNITED STATES BUREAU OF THE CENSUS, 1950. Alphabetical Index of Occupation and Industries. Washington, D.C.: U.S. Government Printing Office.

UNITED STATES BUREAU OF THE CENSUS, 1981. 1980 Census of Populations: Alphabetical Index of Industries and Occupations. Washington, D.C.: U.S. Government Printing Office.

WEGMAN, D. H. \& PETERS, J. M., 1978. Oat cell lung cancer in selected occupations - A case control study. Journal of Occupational Medicine, 20:793796.

WHO (World Health Organization), 1948. Manual of the International Statistics Classification of Diseases, Injuries, and Causes of Death. 6th Revision. Genève: WHO. 